\title{
Tunable and Sensitive Biophotonic Waveguides Based on Photonic-Bandgap Microcavities
}

\author{
Ching Eng Png, Soon Thor Lim, Er Ping Li, Senior Member, IEEE, and Graham T. Reed
}

\begin{abstract}
This paper presents a theoretical study of the singlemode and birefriengence condition of the silicon photonic wires using the imaginary distance beam propagation method. The photonic wires are suitable for integration with active one-dimensional silicon photonic bandgap waveguides. This inherently will reduce the propagation loss caused by the scattering factors within the photonic bandgap structure itself. To the best of our knowledge, we provide for the first time, a systematic study of the various physical parameters that can affect the $Q$-factor and transmission properties in such waveguides. In order to make this technology viable, the waveguides must be tunable, have low attenuation, possess high $Q$-factor, and can be switched. Can these be achieved simultaneously without changing the device width and height dimensions? Furthermore, can we meet these aims without placing unrealistic demands in fabrication? The electrical switching of this device is implemented using a p-i-n optical diode. The diode is predicted to require an ON state power of $81 \mathrm{nW}$ with rise and fall times of 0.2 and $0.043 \mathrm{~ns}$, respectively. The length of the microcavity and the diameter of the air holes are finely tuned with reference to the $Q$-factor and transmission. It will be shown that for certain desired resonant wavelength, the $Q$-factor and transmission properties can be optimized by tuning the length of the cavity and the diameter of the two inner most air holes. This method allows ease of fabrication by not having to vary the waveguide width and height to obtain the tuning effects. Optical simulation was performed using the three-dimensional finite-difference time-domain simulation method.
\end{abstract}

Index Terms-Birefringence, phase modulation, photonic bandgap, silicon-on-insulator (SOI), silicon photonics, single mode.

\section{INTRODUCTION}

$\mathbf{R}$ ECENT development of silicon optical waveguides is moving towards a relatively small scale, typically hundreds of nanometres. Single-mode condition for such waveguides is possible without the need to configure the waveguiding structure into a ridge formation; however, the question arises here, therefore, is the possibility of maintaining the polarization independence property [1]. The use of photonic wire, for example, with height of $400 \mathrm{~nm}$ and width of $400 \mathrm{~nm}$ is suitable for integration with photonic crystal structures [2]. This inherently reduced the propagation loss of the device due

Manuscript received January 27, 2006; revised May 9, 2006. The review of this paper was arranged by Associate Editor R. Lake.

C. E. Png, S. T. Lim, and E. P. Li are with the Institute of High Performance Computing, Agency of Science and Technology, Singapore 117528, Singapore (e-mail: pngce@ihpc.a-star.edu.sg).

G. T. Reed is with the Advanced Technology Institute, University of Surrey, Guildford GU2 7XH, U.K

Color versions of Figs. 1-3, 5, and 6 are available at http://ieeexplore.ieee.org. Digital Object Identifier 10.1109/TNANO.2006.881275 to the sheer amount of scattering occurring within the photonic crystal structure itself.

Photonic bandgap structures have the ability to confine light to small volumes, on the order of $(\lambda / 2 n)^{3}$, where $\lambda$ is the photon wavelength and $n$ is the refractive index of the host material [2]-[5]. The local defect inside the photonic crystal leads to the highly confined optical state. The defect can be in three- (3-D), two- (2-D), or one-dimensional (1-D) photonic crystals. In order to relax fabrication restrictions, the defect in the 1-D photonic crystal is a more attractive way to obtain strong optical confinement. The mechanism for this 1-D photonic crystal is that both the periodical photonic crystal and the high-low index contrast contribute to the photonic confinement. One-dimensional photonic crystals based on waveguide have been fabricated in $\mathrm{Si}-\mathrm{SiO}_{2}$ [2] and $\mathrm{GaAs}-\mathrm{Al}_{x} \mathrm{O}_{y}$ compound semiconductor materials [3]. An air-bridge structure was proposed to improve both the $Q$-factor and transmission performance. Compared with the $Q$-factor of 200 and transmission of 0.72 , the air-bridge structure can provide a $Q$-factor of 320 and transmission of 0.94 [3].

In this paper, we investigate a theoretical study of the photonic wires, to form a concrete foundation on such a structure which allows the occurrence of both single-mode and zero birefringence condition (ZBC) simultaneously using the imaginary distance beam propagation method (IDBPM). We also provide an alternative way to the air-bridge within the photonic crystal structure, to prevent the through substrate wave-leakage; furthermore, the diameter of the inner most air hole is reduced and the cavity length is tuned to obtain an optimized result. An active element is incorporated into the cavity center of the waveguide by way of an optical phase modulator.

\section{Silicon-On-Insulator (SOI) Photonic Wires}

The effect of polarization on photonic wires is based on modal analysis using (IDBPM) [6], [7], a technique based on full-vectorial scheme, and has been successfully employed to investigate the effective index of the fundamental mode in the SOI photonic wire structure. It is worth mentioning that the IDBPM is not the same as the common technique of performing a standard propagation and waiting for the solution to reach steady state. This technique is to provide the option for successively computing the eigenmodes from the fundamental to the higher order modes or even the leaky modes, thus ideal for the solution of mode solving which is much more robust and efficient than the standard BPM. Under the usual IDBPM assumption, the choice of the initial field can help facilitate excitation of the interested guided modes and minimize computation time. An initial symmetric Gaussian field is the most efficient for the computation 


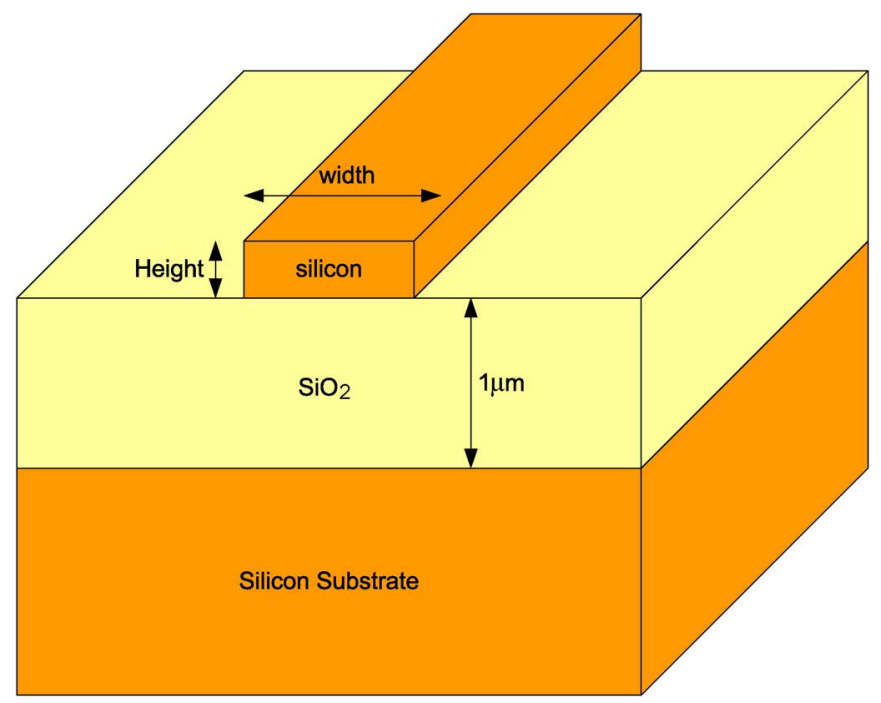

Fig. 1. SOI photonic wire structure.

of the fundamental mode. Thus, such fields can be expanded in the modes of the structure by [7]

$$
\psi_{\text {in }}(x, y, 0)=\sum_{m} c_{m} \psi_{m}(x, y)
$$

The propagation of the field in the $z$-direction can then be expressed as

$$
\psi(x, y, z)=\sum_{m} c_{m} \psi_{m}(x, y) \exp \left[-i\left(n_{m}-n_{o}\right) k z\right]
$$

where $c_{m}$ is a constant. By substituting the physical $z$ axis by $i \tau$ in (2), we obtained

$$
\psi(x, y, \tau)=\sum_{m} c_{m} \psi_{m}(x, y) \exp \left[-i\left(n_{m}-n_{o}\right) k \tau\right] .
$$

Hence, the calculated effective index of the fundamental mode is essentially a function of $\tau$ expressed by

$$
\begin{aligned}
n_{f}(\tau)=n_{o}+\{ & \left(\operatorname{In}\left[\iint \psi(x, y, \tau+\Delta \tau) d x d y\right]\right. \\
& \left.\left.-\operatorname{In}\left[\iint \psi(x, y, \tau) d x d y\right]\right) / k \Delta \tau\right\}
\end{aligned}
$$

where $\Delta \tau$ is the propagation step size, $n_{f}$ is the effective index of the waveguide, $n_{o}$ is utilized reference index, and $n_{m}$ is the effective index of the $m$ th mode the effective index. Hence, (4) will lead to a converged effective index when the modal field reaches the steady states.

The ZBC simulation was set up using the waveguide structure as shown in Fig. 1, which is an SOI channel waveguide or referred to as photonic wire. The refractive indexes for silicon (Si) and silicon dioxide $\left(\mathrm{SiO}_{2}\right)$ are set at 1.444 and 3.477, respectively. Photonic wires with an overall height of 300, 400, 500 , and $600 \mathrm{~nm}$ are analyzed at a wavelength of $1.55 \mu \mathrm{m}$. A similar assumption is made with regard to this simulation as discussed by Chan et al. [8]. In the simulation, a transverse computation window of 2.02 and 2.12 in the $x$ and $y$ direction is utilized. The mesh grid for the $x, y$, and $z$ direction are

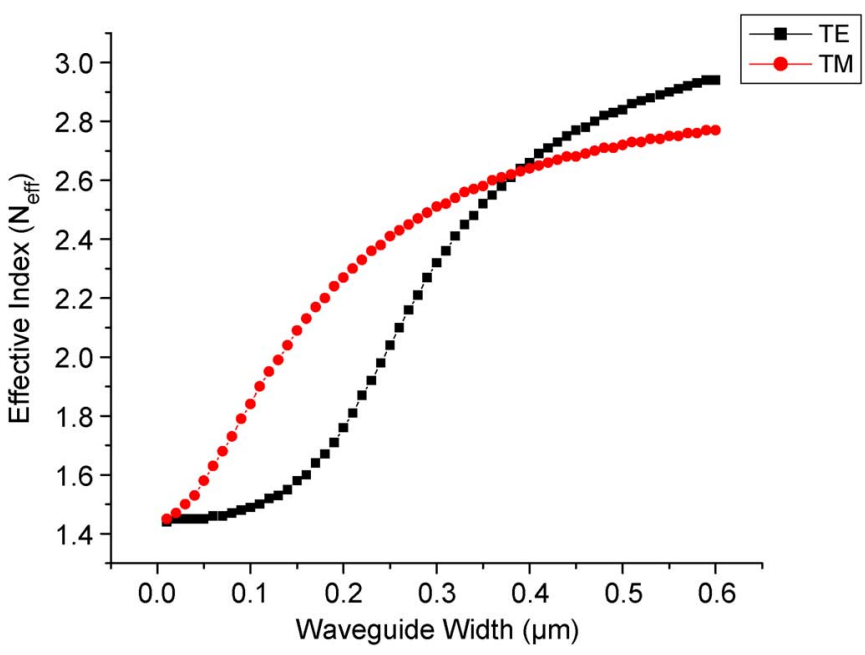

Fig. 2. TE/TM fundamental mode effective indexes for photonic wire of height $400 \mathrm{~nm}$.

$0.005,0.005$, and 0.0005 , respectively. The inherently high refractive index contrast in SOI material enables the realization of photonic wires without sacrificing the mode confinement and single-mode operation.

The increased polarization dependence in small waveguides is derived from the increasingly differing mode shapes of the transverse electric (TE) and transverse magnetic (TM) modes. As we follow the approach for our submicron dimension rib structure as discussed in [8] and extend the analysis to the photonic wire structure with appropriate simulation technique, we can produce a graphical variation of the TE/TM fundamental mode effective indexes as shown in Fig. 2 using photonic wire height of $400 \mathrm{~nm}$ as an example.

Fig. 2 presents the convergence behavior of the calculated effective indexes for both TE and TM modes versus various waveguide widths. It can be noted that the effective indexes increasing with width for a fixed waveguide height. The intersection of both TE and TM mode effective indexes indicated a possible solution for ZBC for a width of $400 \mathrm{~nm}$ and height of $400 \mathrm{~nm}$. The ZBC condition is defined as the difference between the fundamental TE and TM modes. We also produce a graphical presentation of ZBC conditions for different waveguide height and width of interests. Fig. 3 shows various curves of waveguide width against the effective-index difference, each for a different height. By properly selecting the appropriate structure where the curves cross the zero-birefringence axis when the effective indexes of both polarization modes is the same, indicates a possibility to produce ZBC waveguides which can complement our photonic bandgap structure discussed in Section III.

\section{Theoretical Model of 1-D PHOtonic BANDGAP STRUCTURE}

The material system considered here is based upon SOI platform due to its low optical loss and well understood material properties (e.g., [9]). The waveguide is based on a 1-D periodical air hole similar to [2] and is shown in Fig. 4(a). The cavity consists of a Bragg mirror on both sides of the cavity, where $a_{0}$ is the length of the cavity, $a$ is the period of the air holes, $d_{0}$ is 


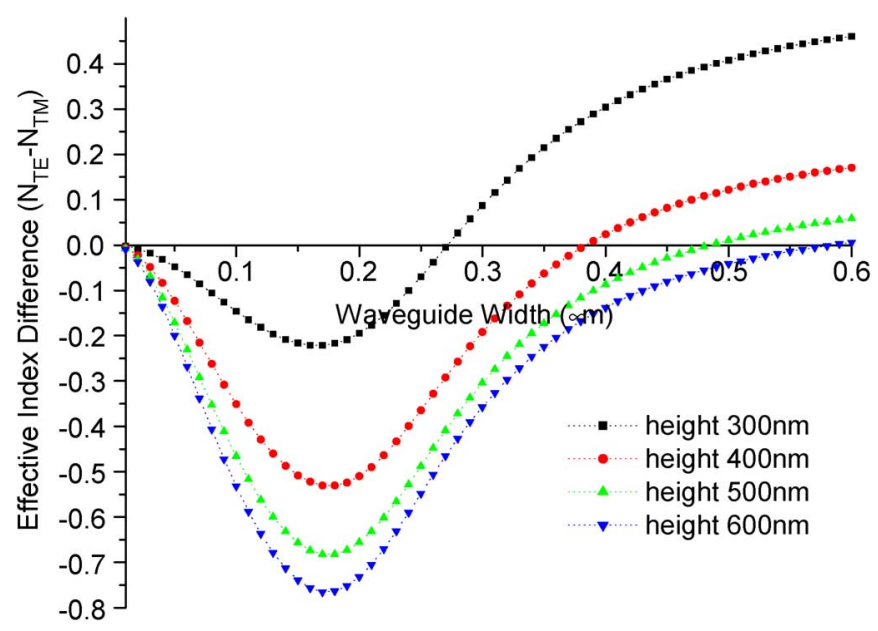

Fig. 3. Effective-index difference calculation using IDBPM for photonic wires with height of $300,400,500$, and $600 \mathrm{~nm}$, respectively.
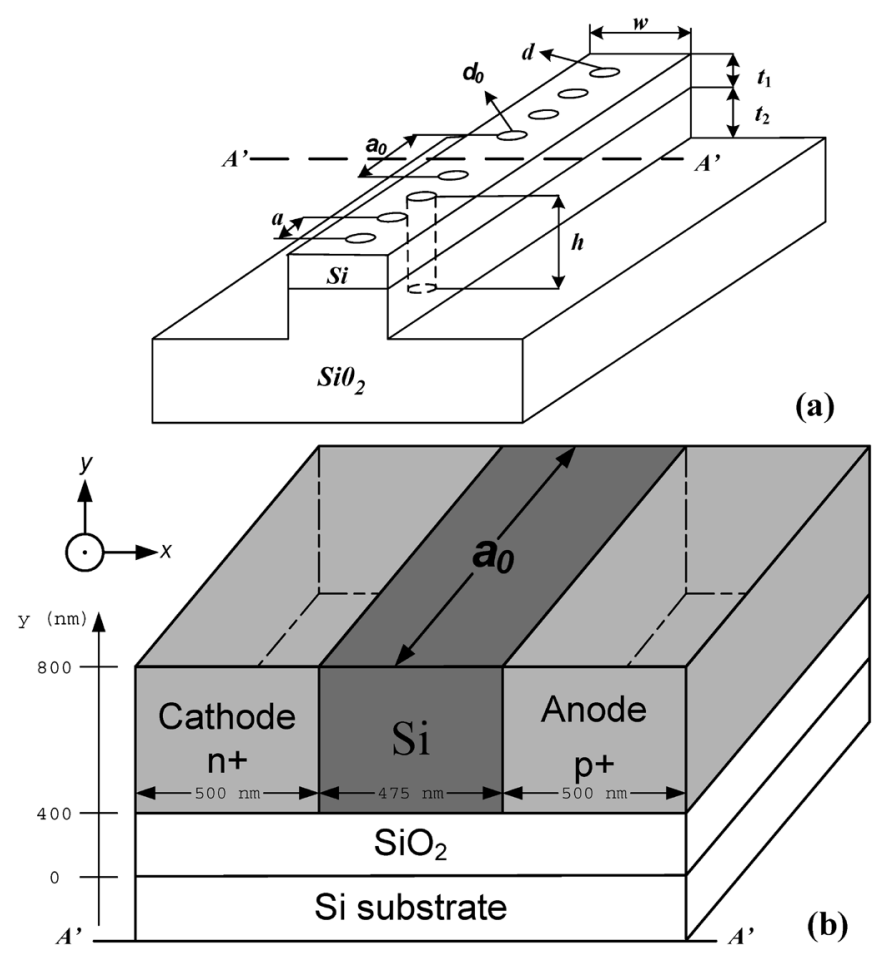

Fig. 4. (a) Photonic crystal waveguide with microcavity with the active device not shown; (b) active switching optical diode in the microcavity region.

the diameter of the two inner most air holes, $d$ is the diameter of the remaining air holes, $w$ is the width of the rib, $t_{1}$ is the thickness of the Si layer, $t_{2}$ is the etching depth of the $\mathrm{SiO}_{2}$ substrate, and $h$ is the etching depth of the air holes. The air holes are etched into the $\mathrm{SiO}_{2}$ substrate, which is different from [2] and [3]. This etch-down design has been adopted to improve the transmission property in photonic crystal slab [10]. The center of this waveguide contains a defect cavity and the active p-i-n diode, shown in Fig. 4(b). The active diode offers switching capability via the free carrier injection effect (described in the optical model below). The device structure of the phase modulator is shown in Fig. 4(b). It is a lateral optical phase modulator integrated into a low loss SOI strip waveguide. The device is a two-terminal $\mathrm{p}-\mathrm{i}-\mathrm{n}$ structure where both $\mathrm{n}$ and $\mathrm{p}$ regions were modeled as highly doped regions with constant doping concentrations of $10^{20} \mathrm{~cm}^{-3}$, and are based around an overall silicon thickness of $400 \mathrm{~nm}$, strip waveguide with a width of $475 \mathrm{~nm}$.

\section{A. Optical and Electrical Modes}

1) Optical Model: Fig. 4(a) depicts the device with air holes etched into the $\mathrm{SiO}_{2}$ substrate. This "etch-down" effect to the substrate was found to be similar in function to the air-bridge design wherein both of them can prevent the wave leaking into the substrate. Furthermore, the etch-down design is simpler from the fabrication point of view. A 3-D finite-difference time-domain (FDTD) package from CST Microwave Studio ${ }^{1}$ was used to simulate the transmission behavior of the photonic crystal structure. Initial simulation validation was performed against the results of [2], where we obtained close agreement with respect to the reported experimental data. With the electrical model described below, the injected electron and hole concentrations at any point of the $\mathrm{p}-\mathrm{i}-\mathrm{n}$ structure can be predicted. Soref and Bennett produced the following expressions relating the refractive index coefficient $(n)$ and absorption coefficient $(\alpha)$ changes in silicon [11] due to the plasma effect, i.e., injection or depletion of free carriers in silicon [12] at a wavelength of $\lambda=1.55 \mu \mathrm{m}$, which are now widely used

$$
\begin{aligned}
\Delta n & =\Delta n_{e}+\Delta n_{h} \\
& =-8.8 \times 10^{-22}\left(\Delta N_{e}\right)-8.5 \times 10^{-18}\left(\Delta N_{h}\right)^{0.8} \\
\Delta \alpha & =\Delta \alpha_{e}+\Delta \alpha_{h} \\
& =8.5 \times 10^{-18}\left(\Delta N_{e}\right)+6.0 \times 10^{-18}\left(\Delta N_{h}\right)
\end{aligned}
$$

where $\Delta n_{e}$ is the refractive index change due to change in free electron concentrations; $\Delta n_{h}$ is the refractive index change due to change in free hole concentrations; $\Delta N_{e}\left(\mathrm{~cm}^{-3}\right)$ is the electron concentration change; $\Delta N_{h}\left(\mathrm{~cm}^{-3}\right)$ is the hole concentration change; $\Delta \alpha_{e}\left(\mathrm{~cm}^{-1}\right)$ is the absorption coefficient variation due to $\Delta N_{e}$; and $\Delta \alpha_{h}\left(\mathrm{~cm}^{-1}\right)$ is the absorption coefficient variation due to $\Delta N_{h}$.

2) Electrical Model: The 2-D ATLAS device simulation package from SILVACO $^{2}$ has been used to predict the dc and transient characteristics of the modulator. The simulator numerically predicts internal physics and device characteristics of semiconductor devices by solving Poisson's equation and the charge continuity equations for holes and electrons. The software allows a complete statistical approach (Fermi-Dirac statistics) when instances such as heavily doped regions are considered. Shockley-Reed-Hall (SRH), Auger, and surface recombination models were included to account for carrier recombination. A carrier concentration dependent SRH recombination model was employed, with an estimated carrier lifetime in the intrinsic $\mathrm{Si}$ device layer (concentration of $10^{15} \mathrm{~cm}^{-3}$ ) of electrons and holes of $\tau_{n}=700 \mathrm{~ns}$ and $\tau_{p}=300 \mathrm{~ns}$, respectively. Contact pads were assumed to be ohmic in nature and hence carry no additional contact resistance or capacitance. From the dc and transient simulations, ATLAS calculates the injected free carrier concentrations in the intrinsic region of the devices for both dc and transient biasing

\footnotetext{
${ }^{1}$ [Online]. Available: http://www.cst.com

${ }^{2}$ SILVACO International, Santa Clara, CA.
} 
TABLE I

SimUlation PARAMETER SETTINGS AT $300 \mathrm{~K}$

\begin{tabular}{|c|c|}
\hline$\lambda(\mu \mathrm{m})$ & 1.55 \\
\hline $\mathrm{Si}$ refractive index & 3.47 \\
\hline $\mathrm{SiO}_{2}$ refractive index & 1.44 \\
\hline Electron carrier lifetime $(\mathrm{ns})$ & 700 \\
\hline Hole carrier lifetime $(\mathrm{ns})$ & 300 \\
\hline $\mathrm{Si}$ background carrier concentration $\left(\mathrm{cm}^{-3}\right)$ & $10^{15}$ \\
\hline
\end{tabular}

conditions. The change in concentration of free carriers is then converted to refractive index change in the device by using (5). This approach has been validated in the past by various authors (e.g., [13]-[15]). In order to maximize this change, we must optimize the interaction between the injected free carriers and the propagating optical mode. The main parameters used in the simulation are shown in Table I.

Following the work of Png et al. [15], it was determined that a high degree of uniformity existed in the predicted injected carrier concentration at the injection levels of interest, throughout the central guiding region of the device. This results in a uniform refractive index change across the waveguiding region. After obtaining the mean value of the injected carrier concentration in the guiding region, we applied the results to (5) and (6) to obtain the resulting changes in refractive index and absorption for the device under investigation at a wavelength of $\lambda=1.55 \mu \mathrm{m}$. Alternatively, the data from the electrical simulation could be transferred to a similar grid-based optical simulator to predict absorption and refractive index changes, but due to the uniform nature of the injected charge, there should be negligible difference between these approaches. The change in refractive index results in a phase shift $\Delta \phi$ in the optical mode given approximately by

$$
\Delta \phi \approx(2 \pi \Delta n L) / \lambda .
$$

Alternatively, we can rearrange (7) for $\Delta n$ and determine the required refractive index change and hence the required carrier density to achieve the desired amount of phase shift for a certain device length or vice-versa. Unless otherwise stated, the active modulator length is assumed to be $275 \mathrm{~nm}$.

\section{B. Results and Discussions}

1) Optical Characteristics: A series of etch-down simulations were performed. We began by studying the transmission property for the structure shown in Fig. 4(a), where the air hole etching depth $h$ takes 400 and $550 \mathrm{~nm}$. We found that $h$ up to $400 \mathrm{~nm}$ can improve the $Q$-factor to around 320, and further increasing $h$ will not provide any obvious improvement. The power transmission at resonance is almost unchanged when the etching depth $h$ takes 400 and $550 \mathrm{~nm}$. Therefore, in the following simulations, all the air holes are etched $400 \mathrm{~nm}$ deep, directly down to the substrate. The radius of the inner-most air holes $d_{0}$ takes $60,70,80,90 \mathrm{~nm}$, respectively, while the cavity length $a_{0}$ takes $275,285,295,305,315 \mathrm{~nm}$, respectively. Fig. 5 shows the effect of $a_{0}$ on both the transmission characteristics and the $Q$-factor. From Fig. 5, we can see that the $Q$-factor decreases when the cavity length $a_{0}$ increases. This is because the optical mode is not as well confined in the cavity when $a_{0}$ is bigger. The $Q$-factor also reduces when the diameter of the

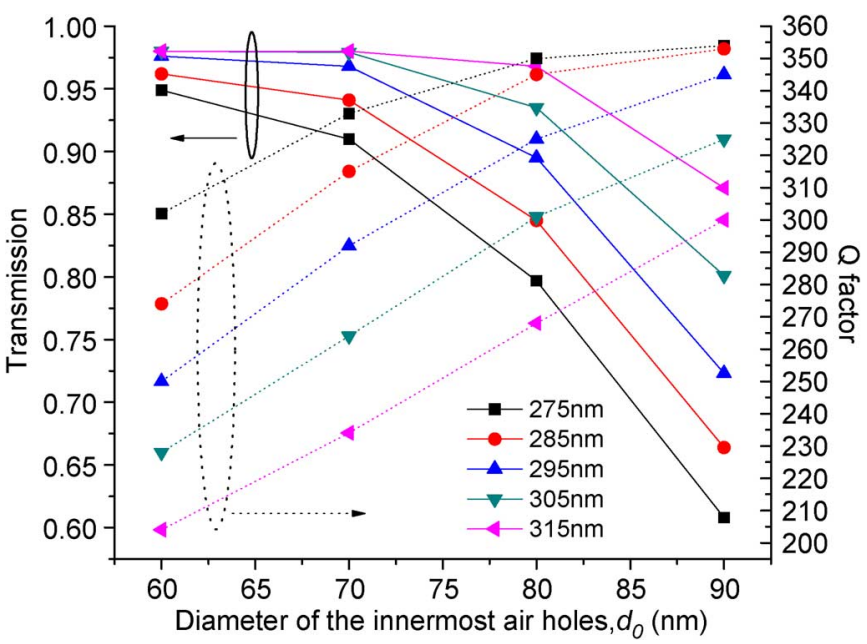

Fig. 5. Transmission and $Q$-factor versus diameter of the innermost air hole $d_{0}$ for various cavity lengths $\left(a_{0}\right)$.

two innermost air holes $d_{0}$ is reduced. The transmission and $Q$-factor characteristics share an inverse relationship. Transmission characteristics improve when $a_{0}$ is increased. When $d_{0}$ is reduced, the optical throughput also increases. What is more important is the fact that a range of device parameters can be chosen to obtain high $Q$ and high transmission properties. For example, when $a_{0}=295 \mathrm{~nm}$ and $d_{0}=80 \mathrm{~nm}$, we obtain $Q \approx 90 \%$ and transmission $(T) \approx 325$. Another example would be when $a_{0}=275 \mathrm{~nm}$ and $d_{0}=70 \mathrm{~nm}$, we obtain $Q \approx 91 \%$ and transmission $(T) \approx 333$. Table II summarizes these results.

The next issue to be addressed is whether the photonic waveguide optical characteristics such as transmission, $Q$-factor, and resonant wavelength can be varied without an active element. Fig. 6 shows the relationship between the resonant wavelength and $d_{0}$ for various values of $a_{0}$ and clearly demonstrates that the optical characteristics can be changed without varying the width and height of the waveguide. Without requiring stringent fabrication restrictions and the absence of an active element to achieve tuning, the deployment of such devices is very attractive. Furthermore, we note a shift of resonant wavelength when changing the cavity length from Fig. 6 . This implies that we can obtain an optimized $Q$ and transmission at a desired resonant wavelength with a range of parameters.

These flexibilities can aid the study of DNA, RNA, proteins, and other biomolecules, where there exists a continual need for the development of environmental, health safety, and clinical microfabricated biosensors. Such a biosensor requires low cost, smaller sample volumes, massive parallelism, and ultrahigh sensitivity [16]-[18]. Many biosensing systems depend on binding of the analyte to individual label particles such as quantum dots, gold particles, and fluorescent dye molecules. These systems are often too complex and large or insufficiently sensitive [19]-[21]. Previous work on 1-D silicon photonic bandgap waveguides have resulted in $Q$-factors at 190 [22] and 265 [2]. These did not evaluate the manufacturability of such devices, a fact which we rectified above. By having a range of device variations, these devices may be suitable for bio-photonic applications where high resolution spectroscopic interrogation is required. 
TABLE II

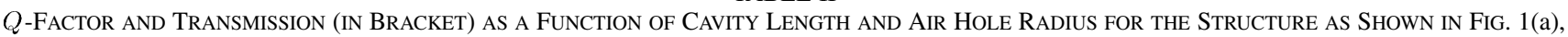
WHERE $a=420 \mathrm{~nm}, t_{1}=200 \mathrm{~nm}, t_{2}=350 \mathrm{~nm}, w=470 \mathrm{~nm}, d=100 \mathrm{~nm}, h=400 \mathrm{~nm}, a_{0}$ AND $d_{0}$ ARE TUNED

\begin{tabular}{|c|c|c|c|c|c|}
\hline$a_{0}$ & $275 \mathrm{~nm}$ & $285 \mathrm{~nm}$ & $295 \mathrm{~nm}$ & $305 \mathrm{~nm}$ & $315 \mathrm{~nm}$ \\
\hline $60 \mathrm{~nm}$ & $302(95 \%)$ & $\begin{array}{c}274 \\
(96 \%)\end{array}$ & $\begin{array}{c}250 \\
(98 \%)\end{array}$ & $\begin{array}{c}228 \\
(98 \%)\end{array}$ & $\begin{array}{c}204 \\
(98 \%)\end{array}$ \\
\hline $70 \mathrm{~nm}$ & 333 & 315 & 292 & 264 & 234 \\
& $(91 \%)$ & $(94 \%)$ & $(90 \%)$ & $(98 \%)$ & $(98 \%)$ \\
\hline $80 \mathrm{~nm}$ & 350 & 345 & 325 & 301 & 268 \\
& $(80 \%)$ & $(85 \%)$ & $(90 \%)$ & $(94 \%)$ & $(97 \%)$ \\
\hline $90 \mathrm{~nm}$ & 354 & 354 & 345 & 325 & 300 \\
& $(61 \%)$ & $(66 \%)$ & $(72 \%)$ & $(80 \%)$ & $(87 \%)$ \\
\hline
\end{tabular}

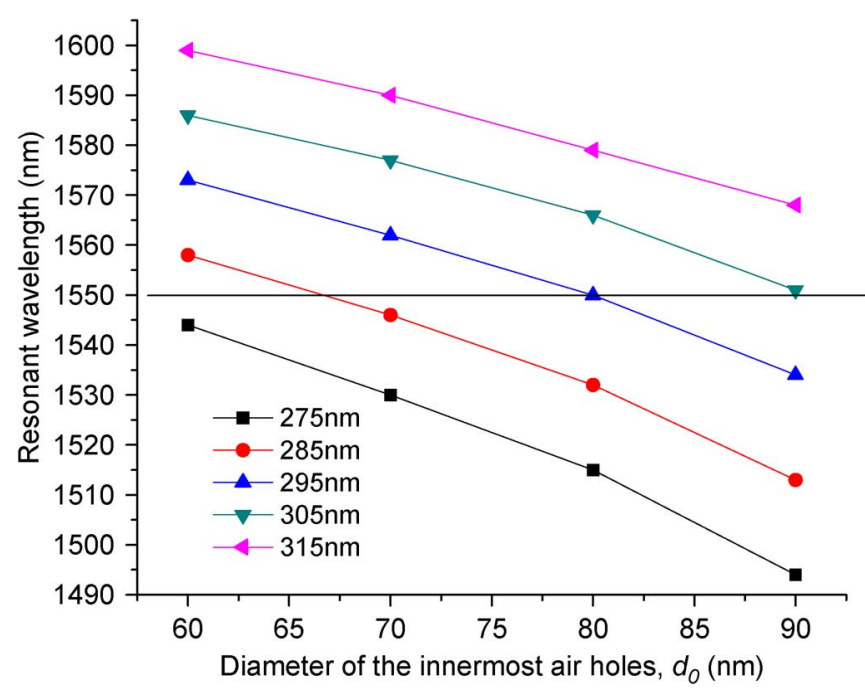

Fig. 6. Resonant wavelength versus the diameter of the innermost air hole $d_{0}$ for various cavity lengths $\left(a_{0}\right)$.

The cavity length $a_{0}$ as proposed in this work is sufficiently large to provide adequate surface for biomolecular interaction. Such interaction depends on the bio-complexes which includes antibody-antigen interaction [23], [24], enzyme-substrate interaction [25], [26] and lectin-glycoprotein interaction [27], etc. In principle, these biomolecular interactions will cause a change in refractive-index of the cavity region, hence altering the original optical spectrum of the 1-D bandgap microcavity in terms of resonating wavelength and/or power reduction. Therefore, if spectral comparison is made between a device with and without a biomolecular sample, an obvious difference will occur. Such mechanisms could be featured into "go/no-go" biosensing instrumentation for initial investigation.

The waveguide device proposed here has a submicrometer height $(400 \mathrm{~nm})$. At such levels, high scattering loss is dominated by the waveguide sidewall roughness and must be addressed. Sakai et al. [28] and Lee et al. [29] have studied the relationship between waveguide losses and waveguide dimensions extensively to enable the design and fabrication of waveguide in SOI with minimal loss, where $0.1-\mathrm{dB} / \mathrm{cm}$ transmission loss was demonstrated for a device that is $200 \mathrm{~nm}$ in Si height [29]. This reaffirms that our proposed device is feasible. The results reported here pertain to TE polarization. The drawback of using strip waveguides is that it is almost impossible to achieve a polarization-independent device where the effective refractive

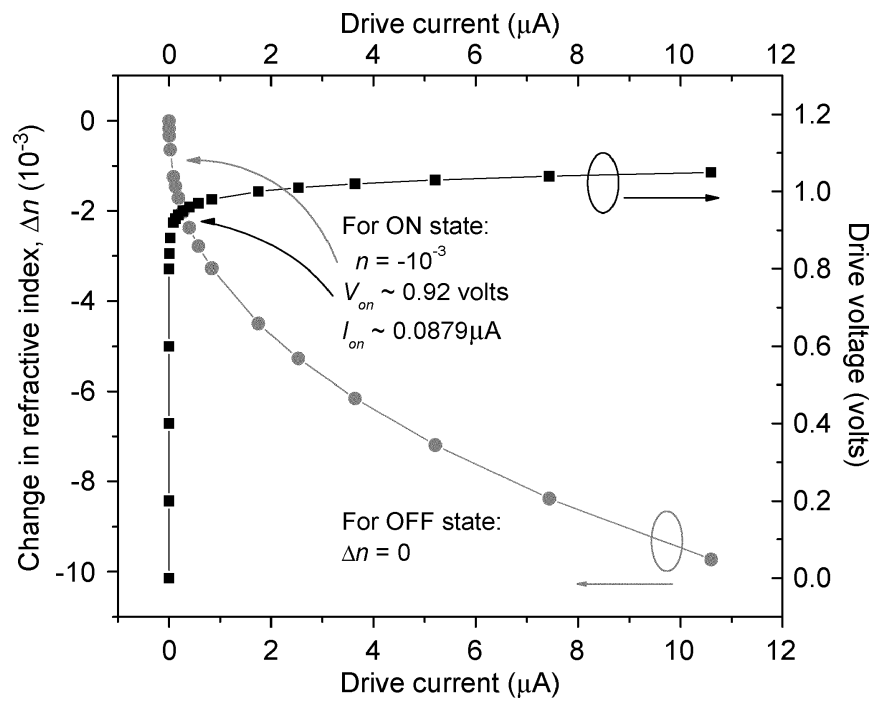

Fig. 7. Predicted relationship between the change in refractive index and drive current along with the voltage characteristics of the optical diode. Pon $\approx 80.9 \mathrm{nW}$.

indexes of TE and TM polarizations match. For a more detailed review of polarizations in waveguides, see, for example, [30].

2) Electrical Characteristics:

a) Static performance: From Fig. 7, the change in refractive index, and hence phase change, varies nonlinearly with applied current (gray line). One factor which contributes to the nonlinearity of the change in phase versus current density relation is the sublinear dependence of the change in free holes $\Delta N_{h}$ with the change in refractive index, as shown in (5). Also, as the modulator is driven harder, more free carriers are injected into the intrinsic region of the device. This increase in the concentration of the previously intrinsic region results in an increase in the Auger recombination rate (at injected carrier concentrations much greater than $10^{17} \mathrm{~cm}^{-3}$ the Auger recombination becomes the dominant recombination process, e.g., [13]). This results in a reduced lifetime in this region and hence we have to drive the modulator harder to achieve an equivalent refractive index change than at lower drive powers. Of course an increase in the recombination rate will result in a faster switching device, i.e., reduction in the rise and fall times of the modulator. The associated current-voltage $(I-V)$ characteristics of the optical diode (black line) are included. Of special interest is the ON state of $N_{e}=N_{h} \approx 3 \times 10^{17} \mathrm{~cm}^{-3}$, which in turn translates 


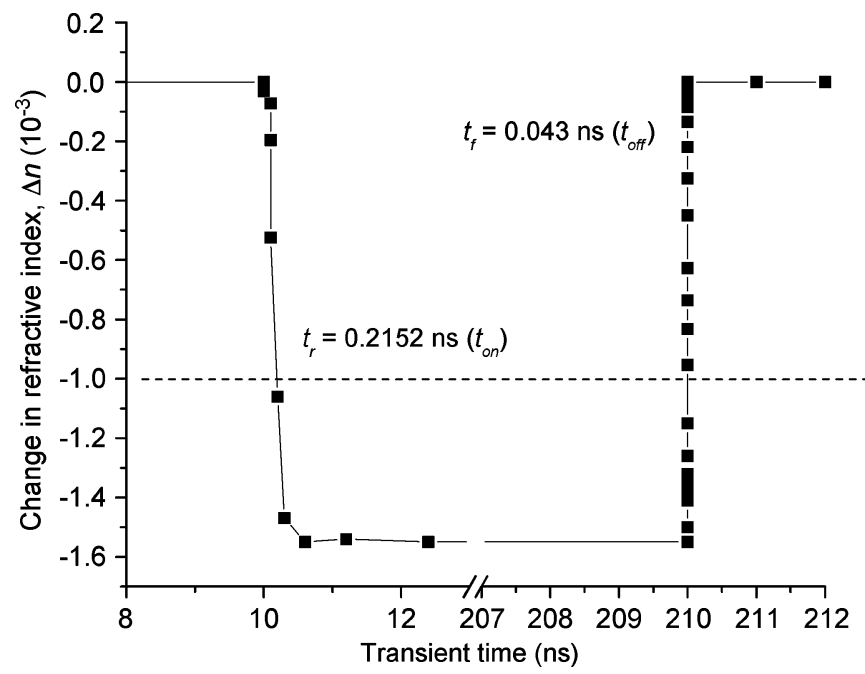

Fig. 8. Predicted transient solution for the optical diode in Fig. 4(b). The rise time is the slower of the two switching times, and hence the limiting factor.

to a change in refractive index of $10^{-3}$, and the OFF state corresponds to no carrier injection state, i.e., no carrier injection. From Fig. 7, the current and voltage required to achieve the ON state are $0.92 \mathrm{~V}$ and $0.0879 \mu \mathrm{A}$, respectively. This corresponds to a power of $\sim 80.9 \mathrm{nW}$.

b) Dynamic performance: For the switching speed, a transient modeling solution was employed. Both anode and cathode were first zero biased for $10 \mathrm{~ns}$, followed by a step increase to $V_{\pi}$ for $200 \mathrm{~ns}$, and a subsequent step decrease to $0 \mathrm{~V}$. $V_{\pi}$ is the voltage corresponding to $180^{\circ}$ phase shift. The rise time $t_{r}$ is defined as the time required for the induced phase shift to change from $10 \%$ to $90 \%$ of the maximum value. Likewise, the fall time $t_{f}$ is defined as the time required for the induced phase shift to change from $90 \%$ to $10 \%$ of the maximum value. For the modulator shown in Fig. 4(b), the rise and fall times were determined to be $t_{r}=0.215 \mathrm{~ns}$ and $t_{f}=0.043 \mathrm{~ns}$, respectively. Fig. 8 shows the rise and fall times and it is clear that the rise time is the slower of the two, and hence, the limiting transition. In order to illustrate clearly both the rise and fall times, a "break" in the horizontal time axis was made so that the rising and falling edge of the transient waveform could be highlighted.

The dynamic optical absorption introduced by switching the device (ON state) corresponds to $N_{e}=N_{h} \approx 3 \times 10^{17} \mathrm{~cm}^{-3}$. Using (6), this injection of both electrons and holes translates to an additional absorption loss of $\sim 4.35 \mathrm{~cm}^{-1}$ (i.e., $18.9 \mathrm{~dB} / \mathrm{cm}$ ). This would result in a dynamic optical absorption of approximately $0.0005 \mathrm{~dB}$ if the active device length is $275 \mathrm{~nm}$. It should be noted that the optical attenuation could be improved by optimizing the position of the anode and cathode dopant contact windows.

The modulator device speed reported here is rather modest and nowhere near one of the fastest reported recently [31]. Nonetheless, the device switching performance can be improved without changing its physical dimensions by overdriving during the device rise and fall times [15]. This method was recently employed by Xu et al. [32] to overdrive a strip-based ring resonator waveguide to operate at a data rate of $1.5 \mathrm{~Gb} / \mathrm{s}$. However, this is at the expense of increasing the device complexity.

\section{CONCLUSION}

Strip-based photonic crystal microcavity devices are studied using the 3-D FDTD method. The air holes are etched down to the substrate to prevent wave leakage. The $Q$-factor, resonant wavelength, and transmission characteristics are of particular interest.

We demonstrated via simulation that high $Q$-factor, high transmission characteristics $(>90 \%)$, and the ability to tune the resonant wavelength can be achieved without modifying the width and height of the photonic crystal waveguide. This was attained by tuning the cavity length and radius of the innermost air holes. A range of parameters capable of meeting these requirements was identified, which in turn, relaxes the demands on fabrication and make these devices more attractive for commercial deployment in bio-photonic and optical interconnect applications.

Improvement of over $30 \%$ in $Q$-factor was achieved when compared to results in [2] (354 versus 265). This device can be switched by implementing an optical modulator in the cavity region. The characteristics of the optical modulator was analyzed using a 2-D semiconductor simulation package SILVACO to investigate the $\mathrm{dc}$ and transient behavior of the active region of the modulator. The optical device as predicted utilized only $81 \mathrm{nW}$ between the ON and OFF state, with a duration of $\sim 0.2 \mathrm{~ns}$.

\section{ACKNOWLEDGMENT}

The authors would like to thank Dr. S. Jin for discussions.

\section{REFERENCES}

[1] G. T. Reed and A. P. Knights, Silicon Photonics: An Introduction. New York: Wiley, ISBN: 0-470-87034-6.

[2] J. S. Foresi, P. R. Villeneuve, J. Ferrera, E. R. Thoen, G. Steinmeyer, S Fan, J. D. Joannopoulos, L. C. Kimerling, H. I. Smith, and E. P. Ippen, "Phonotic-bandgap mocrocavities in optical waveguides," Nature, vol. 390, pp. 143-145, 1997

[3] D. J. Ripin, K. Y. Lim, G. S. Petrich, P. R. Villeneuve, S. Fan, E. R. Thoen, J. D. Joannopoulos, E. P. Ippen, and L. A. Kolodziejski, "One-dimensional photonic bandgap microcavities for strong optical). confinement in GaAs and $\mathrm{GaAs} / \mathrm{Al}_{x} \mathrm{O}_{y}$ semiconductor waveguides,' J. Lightw. Technol., vol. 17, no. 11, pp. 2152-2160, Nov. 1999.

[4] J. G. Fleming and S. Y. Lin, "Three dimensional photonic crystal with a stop band from 1.35-1.95 $\mu \mathrm{m}$," Opt. Lett., vol. 52, pp. 49-51, 1999.

[5] P. Lalanne, S. Mias, and J. P. Hugonin, "Two physical mechanisms for boosting the quality factor to cavity volume ratio of photonic crystal microcavities," Opt. Express, vol. 12, no. 3, pp. 458-467, 2004.

[6] BeamPROP. Rsoft, Inc. Research Software, Ossininh, NY.

[7] Y. Z. He and F. G. Shi, "Finite-difference imaginary-distance beam propagation method for modelling of fundamental mode of photonic crystal fibers," Opt. Commun., vol. 225, pp. 151-156, 2003.

[8] S. P. Chan, C. E. Png, S. T. Lim, G. T. Reed, and V. M. N. Passaro, "Single mode and polarisation independent silicon-on-insulator waveguides with small cross section," J. Lightw. Technol., vol. 23, no. 6, pp. 2103-2111, Jun. 2005.

[9] A. Rickman, G. T. Reed, B. L. Weiss, and F. Namavar, "Low-loss planar optical waveguides fabricated in SIMOX material," IEEE Photon. Technol. Lett., vol. 4, no. 6, pp. 633-635, Jun. 1992.

[10] G. Böttger, C. Liguda, M. Schmidt, and M. Eich, "Improved transmission characteristics of moderate refractive index contrast photonic crystal slabs," Appl. Phys. Lett., vol. 18, no. 14, pp. 2517-2519, 2002.

[11] R. A. Soref and B. R. Bennett, "Kramers-Kronig analysis of E-O switching in silicon," in SPIE Integrated Opt. Circuit Emg., 1986, vol. 704, pp. 32-37. 
[12] — - "Electrooptical effects in silicon," IEEE J. Quantum Electron., vol. QE-23, no. 1, pp. 123-129, Jan. 1987.

[13] C. K. Tang, G. T. Reed, A. J. Walton, and A. G. Rickman, "Low-loss, single-mode, optical phase modulator in SIMOX material," J. Lightw. Technol., vol. 12, no. 8, pp. 1394-1400, Aug. 1994.

[14] P. D. Hewitt and G. T. Reed, "Improved modulation performance of a silicon p-i-n device by trench isolation," J. Lightw. Technol., vol. 19, no. 3, pp. 387-390, Mar. 2001.

[15] C. E. Png, S. P. Chan, S. T. Lim, and G. T. Reed, "Optical phase modulators for $\mathrm{MHz}$ and $\mathrm{GHz}$ modulation in silicon-on-insulator (SOI)," $J$. Lightw. Technol., vol. 22, no. 6, pp. 1573-1583, Jun. 2004.

[16] F. Vollmer, S. Arnold, D. Braun, I. Teraoka, and A. Libchaber, "Multiplexed DNA quantification by spectroscopic shift of two microsphere cavities," Biophys. J., vol. 85, pp. 1974-1979, 2003.

[17] L. R. Huang, J. O. Tegenfeldt, J. J. Kraeft, J. C. Sturm, R. H. Austin, and E. C. Cos, "A DNA prism for high-speed continuous fractionation of large DNA molecules," Nat. Biotechnol., vol. 20, pp. 1048-1051, 2002.

[18] G. Wu, R. H. Datar, K. M. Hansen, T. Thundat, R. J. Cote, and A. Majumdar, "Bioassay of prostate-specific antigen (PSA) using microcantilevers," Nat. Biotechnol., vol. 19, pp. 856-860, 2001.

[19] A. J. Haes and R. P. Van Duyne, "A nanoscale optical biosensor: real time immunoassay and nanoparticle adhesion," J. Phys. Chem. B, vol. 107, pp. 1772-1780, 2003.

[20] S. Nie and R. Emory, "Probing single molecules and single nanoparticles by surface-enhanced Raman Scattering," Science, vol. 275, pp. 1102-1106, 1997.

[21] V. S.-Y. Lin, K. Moteshari, K.-P. S. Dancil, M. J. Sailor, and M. R. Ghadiri, "A porous silicon-based optical interferometric biosensor," Science, vol. 278, pp. 840-843, 1997.

[22] B. Schmidt, V. Almeida, C. Manolatou, S. Preble, and M. Lipson, "Nanocavity in a silicon waveguide for ultrasensitive nanoparticle detection," Appl. Phys. Lett., vol. 85, pp. 4854-4856, 2004.

[23] S. M. Barnard and D. R. Walt, "Chemical sensors based on controlledrelease polymer systems," Science, vol. 251, pp. 927-929, 1991.

[24] P. S. Stayton, T. Shimoboji, C. Long, A. Chilkoti, G. Ghen, J. M. Harris, and A. S. Hoffman, "Control of protein-ligand recognition using a stimuli-responsive polymer," Nature, vol. 378, pp. 472-474, 1995.

[25] A. Riklin, E. Katz, I. Wiliner, A. Stocker, and A. F. Bückmann, "Improving enzyme-electrode contacts by redox modification of cofactors," Nature, vol. 376, pp. 672-675, 1995.

[26] R. V. Parthasarathy and C. R. Martin, "Synthesis of polymeric microcapsule arrays and their use for enzyme immobilization," Nature, vol. 369, pp. 298-301, 1994.

[27] Y. Shinohara, H. Sota, F. Kim, M. Shimizu, M. Gotoh, M. Tosu, and Y. Hasegawa, "Use of a biosensor based on surface plasmon resonance and biotinyl glycans for analysis of sugar binding specificities of lectins," J. Biochem., vol. 117, pp. 1076-1082, 1995.

[28] A. Sakai, T. Fukazawa, and T. Baba, "Low loss ultra-small branches in a silicon photonic wire waveguide," IEICE Trans. Electron., vol. E85-C, pp. 1033-1038, 2002.

[29] K. K. Lee, D. R. Lim, H. Luan, A. Agarwal, J. Foresi, and L. C. Kimerling, "Effect of size and roughness on light transmission in a $\mathrm{Si} / \mathrm{SiO} 2$," IEICE Trans. Electron., vol. E85-C, pp. 1033-1038, 2002.

[30] G. T. Reed, G. Z. Masanovic, W. R. Headley, B. Timotijevic, F. Y. Gardes, S. P. Chan, P. Waugh, N. G. Emerson, C. E. Png, M. J. Paniccia, A. Liu, D. Hak, and V. M. N. Passaro, "Issues associated with polarization independence in silicon photonics," IEEE J. Quantum Electron., 2006, (invited), to be published.

[31] L. Liao, D. Samara-Rubio, M. Morse, A. Liu, D. Hodge, D. Rubin, U. D. Keil, and T. Franck, "High speed silicon Mach-Zehnder modulator," Opt. Express, vol. 13, pp. 3129-3135, 2005.

[32] Q. Xu, B. Shmidt, S. Pradhan, and M. Lipson, "Micrometre-scale silicon electro-optic modulator," Nature, vol. 435, pp. 325-327, 2005.
Ching Eng Png received the B.Eng. degree in Electronic and Electrical Engineering (with First Class Honours) and the Ph.D. degree from the University of Surrey, U.K., in 1999 and 2004, respectively. His Ph.D. work was supervised by Prof. G. T. Reed and focused on silicon photonics.

From November 1999 to September 2000, he was with Agilent Technologies, Singapore, as a Gigabit Optical Transceiver Engineer. He received scholarships from Bookham Inc. and ORS to pursue his Ph.D. work. He is currently with the Institute of High Performance Computing, A*Star, Singapore, working on bio-photonics and silicon photonics. He is also Adjunct Assistant Professor of Electrical Engineering at the National University of Singapore (NUS).

Dr. Png was awarded the Royal Academy of Engineering Prize and the Institution of Electrical Engineers (IEE) Hudswell International Research Scholarship for his work on silicon optical modulators.

Soon Thor Lim was born in Singapore in 1975. He received the Electrical and Electronic degree (with First Class Honours) and the Ph.D. degree from the University of Surrey, in 2000 and 2005, respectively.

In 2001, he received a scholarship from Agilent Technologies (Singapore) to pursue his doctorate degree under the supervision of Prof. G. T Reed working on silicon photonics with particular emphasis on design and modelling of arrayed waveguide grating (AWG). Currently, he is with the Institute of High Performance Computing, A*Star, Singapore. His research interests include SOI waveguides, photonics crystal, and photonics bio sensing applications.

Er Ping Li (M'93-SM'01) received the M.Sc. degree in electrical engineering from Xi'an Jiaotong University, Xi' an, China, in 1986, and the Ph.D. degree in electrical engineering from Sheffield Hallam University, Sheffield, U.K., in 1992.

He worked as a Research Fellow from 1989 to 1990 and then as a Lecturer from 1991 to 1992 at Sheffield Hallam University, U.K. Between 1993 and 1999, he was a Senior Research Fellow, Principal Engineer, and Technical Manager/Director with the Singapore Research Institute and Industry. Since 2000, he has been with the Institute of High Performance Computing, A*Star, Singapore, where he is currently a Senior Scientist and Senior R\&D Manager of the Computational Electromagnetics and Electronics Division. He has served as Chair of a number of international conferences and is the Technical Advisor to a number of multinational companies in Asia. He has published more than 90 technical papers in international referred journals and conferences and coauthored three book chapters. His research interests include fast and efficient computational electromagnetics, EMC/EMI, high-speed electronic modeling, and computational nanotechnology.

Graham T. Reed joined the University of Surrey in 1989 with the aim of establishing a research activity in guided wave optoelectronics, and now leads an internationally recognized group. He is responsible for initiating a new research field in the U.K. on silicon integrated optical circuits, and his group has produced a series of leading technical advances in the field worldwide, notably in optical modulators, grating couplers, and optical sensing applications. A testament to the originality and potential of the silicon work is that Bookham Technology plc adopted it as their core business in the early 1990s. His work is built upon collaborative arrangements with both industry and academia alike. His work has been associated with companies and universities in the U.K., the U.S., France, Italy, Germany, Japan, and Singapore. He has contributed to more than 150 publications in the field of guided wave optoelectronics, co-authored the first text book on silicon photonics, and contributed to several patents.

Prof. Reed is a Fellow of the IEE. 